\title{
A Sorting Library for FPGA Implementation in OpenCL Programming
}

\author{
Ryohei Kobayashi \\ University of Tsukuba \\ 1-1-1 Tennodai, Tsukuba, Ibaraki \\ 305-8577 Japan \\ kobayashi@cs.tsukuba.ac.jp
}

\author{
Taisuke Boku \\ University of Tsukuba \\ 1-1-1 Tennodai, Tsukuba, Ibaraki \\ 305-8577 Japan
}

\author{
Norihisa Fujita \\ University of Tsukuba \\ 1-1-1 Tennodai, Tsukuba, Ibaraki \\ 305-8577 Japan
}

\begin{abstract}
In this study, we focus on data sorting, which is a basic arithmetic operation, and we present a sorting library that can be used with the OpenCL programming model for fieldprogrammable gate arrays (FPGAs). Our sorting library is built by combining three hardware sorting algorithms. It consumes more than twice the overall hardware resources compared to the merge sort restructured for the OpenCL programming model for FPGAs. However, its operating frequency is $1.09 \mathrm{x}$ higher and its sorting throughput is three orders of magnitude greater than the baseline.
\end{abstract}

\section{KEYWORDS}

FPGA, Sorting, Intel FPGA SDK for OpenCL

\section{ACM Reference Format:}

Ryohei Kobayashi, Kento Miura, Norihisa Fujita, Taisuke Boku, and Toshiyuki Amagasa. 2021. A Sorting Library for FPGA Implementation in OpenCL Programming. In International Symposium on Highly Efficient Accelerators and Reconfigurable Technologies (HEART'21), June 21-23, 2021, Online, Germany. ACM, New York, NY, USA, 6 pages. https://doi.org/10.1145/3468044.3468054

\section{INTRODUCTION}

High-level synthesis (HLS) techniques have emerged and evolved considerably in the last decade for mitigating fieldprogrammable gate array (FPGA) implementation cost. In

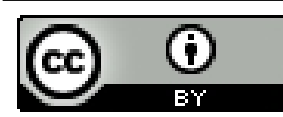

This work is licensed under a Creative Commons Attribution International 4.0 License.

HEART '21, June 21-23, 2021, Online, Germany

(C) 2021 Copyright held by the owner/author(s).

ACM ISBN 978-1-4503-8549-7/21/06.

https://doi.org/10.1145/3468044.3468054

\section{Toshiyuki Amagasa}

University of Tsukuba

1-1-1 Tennodai, Tsukuba, Ibaraki 305-8577 Japan

particular, the Xilinx SDAccel and Intel FPGA SDK for OpenCL are all-in-one development environment that hide from the user the fundamental parts of FPGA implementation, such as external memory controllers, the PCIe interface, and device drivers, enabling implementation of FPGA applications without the user being aware of them. The Intel FPGA SDK for OpenCL provides a mechanism for controlling the peripherals for the FPGA fabric from OpenCL, and we previously proposed a framework [1] using the mechanism to assist application developers in developing HPC applications in the OpenCL programming layer. Therefore, we believe that the Intel FPGA SDK for OpenCL is currently the best solution for application developers in using FPGAs.

However, whereas register transfer level (RTL)-based FPGA implementations allow designers to define to a fine degree the appropriate architecture for their applications, OpenCLbased programming models are limited in their expressiveness. In addition, OpenCL-based programming models often suffer from place and route problems that limit the maximum frequency or entirely prevent a successful synthesis [2]. Therefore, to develop high-performance FPGA applications with low programming cost, it is necessary to optimize general-purpose computation kernels for practical application development for FPGAs and to make them available to users. For example, the authors in [2] proposed an OpenCL implementation of Cannon's matrix multiplication algorithm optimized for Intel Stratix 10 FPGAs, assuming that it could serve as a building block for algorithms that are built upon the base functionality of matrix multiplications.

In this study, we propose a sorting library that can be used with the OpenCL programming model for FPGA. Sorting is a basic arithmetic operation used in the development of various applications, and to accommodate its wide range of uses, libraries are provided in the form of $\operatorname{std}:$ : $\operatorname{sort}()$ for $\mathrm{C}++$ 


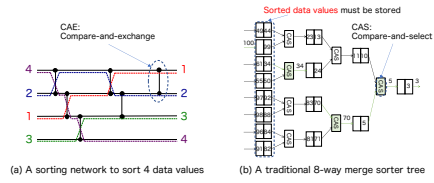

Figure 1: Underlying hardware sorting algorithms: (a) a sorting network and (b) a merge sorter tree.

and thrust: : sort () for CUDA. We develop a hardware engine in RTL to perform the sorting process and call it a library from the OpenCL kernel code. The engine is built by combining the following three hardware sorting algorithms: the sorting network, high-bandwidth merge sorter tree, and virtual merge sorter tree. These algorithms are designed to run at high operating frequencies so that the engine called from the OpenCL kernel does not become the critical path and degrade the overall performance of the application. In addition, the engine's configuration can be set by parameters, and the application developer can determine the best configuration for the sorting engine according to our derived performance model while remaining at the OpenCL abstraction level. We evaluated the performance of our proposed sorting library and determined that its sorting throughput was three orders of magnitude greater than that of the merge sort algorithm restructured for the OpenCL-based implementation.

\section{HARDWARE SORTING ALGORITHM}

Hardware sorting algorithms are hardware models that embody a processing procedure suitable for data sorting by hardware. In particular, a sorting network [3] and merge sorter tree [4] are suitable for FPGAs in terms of parallelism, ease of control, and operating frequency, and numerous related studies that use them have been conducted [5-9]. In this section, we introduce these basic behaviors as a background for understanding our developed sorting engine. To avoid ambiguity in the description, the hardware sorting algorithm described in this study sorts data values with integer keys in ascending order.

The sorting network is a hardware model for sorting sequences of numbers that consist of wires and a compareand-exchange (CAE) unit. Figure 1(a) shows its behavior. The wires are responsible for propagating values, and the CAE unit has two wires for input and output. When two values are input, it outputs the smaller value to one and the larger value to the other. This hardware model can sort the elements in parallel without the need for complex control logic.

The merge sorter tree is a hardware model for merging multiple sorted data values and is composed of a set of compareand-select (CAS) units laid out in a binary tree. The CAS

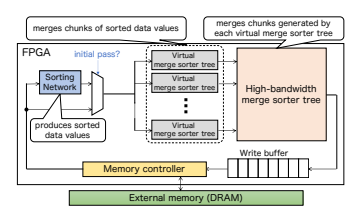

Figure 2: Our developed sorting engine.

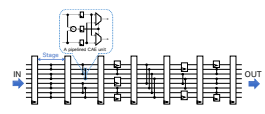

Figure 3: Pipelined Batcher's odd-even sorting network with eight inputs and eight outputs.

unit is a combinational circuit that compares the keys of two input data values and selects one of them according to the comparison result. Figure 1(b) shows a traditional 8-way merge sorter tree and its behavior at a certain clock cycle. At each cycle, the keys are compared in all CAS units, and the data values that are output according to the comparison results are stored in first-in/first-out buffers (FIFOs), and eventually, sorted data values are ejected from the root of the merge sorter tree. FIFOs located at the input port of the tree must always contain sorted data values and, therefore, the sorting network is often used to produce them [5].

\section{FPGA-BASED SORTING ENGINE}

Figure 2 shows our sorting engine that is built by combining three hardware sorting algorithms: the sorting network, high-bandwidth merge sorter tree, and virtual merge sorter tree. In this section, we first explain the mechanism of each hardware sorting algorithm and then describe the sorting engine's behavior.

\subsection{Batcher's Odd-Even Sorting Network}

We use Batcher's odd-even sorting network [10], where Figure 3 shows an example with eight inputs and eight outputs. This sorting network can be implemented as a purely combinational circuit, but its naive implementation causes a performance degradation due to large network delay. To avoid degradation of the operating frequency and to improve the network throughput, this network is commonly implemented as a pipelined circuit by inserting registers between each stage. In addition, the sorting network used in the sorting engine employs a two-stage pipelined CAE unit [6]. This allows the critical path to be only one comparator, thereby increasing the operating frequency.

\subsection{High-bandwidth Merge Sorter Tree}

The traditional merge sorter tree described in Section 2 can output only a maximum of one value per cycle, and at least $n$ 


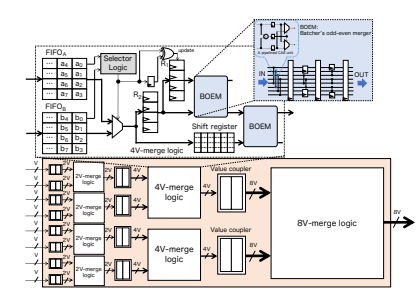

Figure 4: High-bandwidth merge sorter tree [7].

cycles are required to output $n$ values. If the tree can output $m$ values per cycle, the number of cycles required to output all the values can be reduced to a maximum of $\frac{n}{m}$, and several methods to achieve that throughput have been proposed in recent years [5-7]. We call these merge sorter trees high-bandwidth merge sorter trees and incorporate the one proposed in [7] into our sorting engine.

Figure 4 shows a high-bandwidth merge sorter tree proposed in [7]. This tree is built by connecting the merging logic for multiple data values in the form of a binary tree. As the number of input ports of the tree increases, the number of data values output per cycle from the root of the tree increases, as when several tributaries join to form a wide river. In Figure 4, the number of input ports is eight, and incoming eight data streams are merged into a single data stream, which is then output with a throughput of a maximum of eight data values per cycle.

We briefly explain the merging logic underlying this tree's behavior. With the logic of merging four data values taken as an example, it can be seen that two Batcher odd-even mergers (BOEMs) are connected in series. In this manner, the feedback path [5], which is necessary to merge multiple data values constantly, is successfully eliminated, and the logic can be pipelined at a high operating frequency. The most outstanding point of the proposed method in [7] is that it efficiently solves the tie-record issue [6] by developing timelined bundle ordering, in which R1 and R2 are connected in series, and by selecting BOEM as the merger instead of the bitonic merger used in [6]. Because of this mechanism, the tree proposed in [7] exhibited the best merging performance at the stage when we were developing the specification of the sorting engine and, therefore, we decided to adopt it as a component of the engine.

Because the tree in [7] is also a merge sorter tree, increasing the number of input ports not only increases the number of data values output per cycle from the root of the tree, but it also increases the number of sorted data values in the output data stream. However, as the number of input ports of the tree is increased, the hardware resource usage increases linearly. Therefore, the size of the tree that can be implemented in an FPGA is limited to a certain extent, and the number of sorted data values in the output data stream

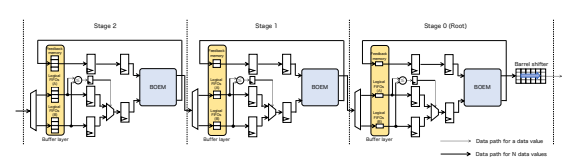

Figure 5: Multi-cycle virtual merge sorter tree.

generated by passing through the tree does not scale. We describe a method to overcome this problem in the section that follows.

\subsection{Virtual Merge Sorter Tree}

In $[8,9]$, the authors proposed hardware-efficient multi-way merge sorter trees that are logically equivalent to the traditional merge sorter tree described in Section 2. We call those trees virtual merge sorter trees and describe their mechanism as follows.

In the traditional merge sorter tree, the CAS unit located at the root selects and outputs only one value on either side at a time. Therefore, in each stage of the tree, only one CAS unit as well as one FIFO dequeue request and one FIFO enqueue request are active. This is highlighted in Figure 1(b). When this characteristic is taken advantage of, the virtual merge sorter tree proposed in $[8,9]$ reduces hardware resource usage from $O(W)$ to $O\left(\log _{2} W\right)$ by placing only one CAS unit in each stage, thus working in a time sharing manner and integrating the FIFOs between CAS units into a single buffer layer implemented in the FPGA's Block RAM, where $W$ is the number of leaves in the tree. In our sorting engine, the virtual merge sorter tree is replicated as many times as the number of ports of the high-throughput merge sorter tree described in Section 3.2, and the output port of each virtual merge sorter tree is connected to each input port of the high-throughput merge sorter tree, which builds a merge sorter tree having both high throughput and a wide input width.

However, the virtual merge sorter trees proposed in $[8,9]$ have the disadvantage whereby their operating frequencies are less than half that of the high-bandwidth merge sorter tree proposed in [7]. Therefore, the performance of the highbandwidth merge sorter tree cannot be maximized by simply connecting the virtual merge sorter tree proposed in $[8,9]$. To solve this problem, we developed a multi-cycle virtual merge sorter tree, as shown in Figure 5.

The developed tree outputs $N$ data values per $N$ cycles, whereas the virtual merge sorter tree proposed in $[8,9]$ outputs only one data value per cycle. To output multiple sorted data values, we replaced the CAS units in each stage with the BOEM used in [7] and added a memory area to the buffer layer to store the data values fed back from the BOEM. Similar to the CAS unit in $[8,9]$, the BOEM works in a time sharing manner. It merges the data read from the logical FIFOs in 
the buffer layer corresponding to the request coming from the upper stage and selected by the selector with the data read from the feedback memory. It then outputs the smaller data values to the upper stage and writes the larger data values back to the corresponding area in the feedback memory. Then, the sorted data values output from the BOEM at the root are extracted as a single data value at a clock cycle by the barrel shifter and input to the high-bandwidth merge sorter tree. Therefore, the number of data values ejected per cycle of the developed tree is the same as that of the virtual merge sorter trees proposed in [8,9]. However, the critical path of the developed tree is only one comparator and, consequently, it can operate at a frequency as high as that of the high-bandwidth merge sorter tree. In addition, the datapath in the BOEM used in the developed tree is optimized by using techniques in [7] such as redundant CAS unit elimination and datapath decoupling for payload, resulting in a hardware-efficient implementation.

\subsection{Sorting Engine Behavior}

Because our design concept of the sorting engine is basically the same as that in [5], it completes the sorting process by performing multiple memory round-trips of the data. In this section, we explain how the sorting engine sorts 2,048 unsorted data values. Here, we assume the width of the sorting network, number of leaves in the virtual merge sorter tree, and number of input ports in the high-bandwidth merge sorter tree to be 8,4 , and 4 , respectively.

Similar to [5], during the initial pass of the sorting process, the data for input can come from anywhere. Therefore, the 2,048 unsorted data values stored in the external memory are read out sequentially by the memory controller and sent to the virtual merge sorter trees through the sorting network. This is because the virtual merge sorter trees expect their inputs to be chunks of sorted data values, and a single chunk contains eight sorted data values at this point. These chunks are merged in each virtual merge sorter tree and then sent to the high-bandwidth merge sorter tree. The merge sorter tree then merges the chunks generated by each virtual merge sorter tree at a data rate of four data values per cycle and enqueues sorted data values output from the highbandwidth merge sorter tree into the write buffer. The data values stored in the write buffer are then sequentially written back to the external memory by the memory controller. The sorting engine uses double buffering to allow both writing and reading from memory simultaneously. In this initial pass, 16 chunks of sorted data values are generated by the sorting engine and written to the external memory. At this point, each chunk contains 128 sorted data values.

On passes after the initial pass, the virtual merge sorter tree must retrieve data from a particular chunk that matches

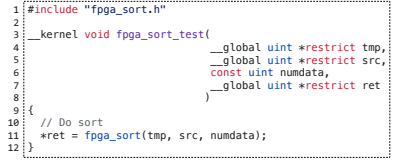

Figure 6: OpenCL kernel code snippet of the data sorting.

the tree input of the request, which is similar to [5]. Therefore, the first four chunks of the 16 chunks are merged in virtual merge sorter tree 0 , and the next four are merged in virtual merge sorter tree 1 . Each virtual merge sorter tree outputs 512 sorted data values, which are merged by the high throughput merge sorter tree. Then, the fully sorted data values are sequentially overwritten back to where initial data values are stored in the external memory.

Let $N_{\text {pass }}$ be the number of passes. It can be formulated as $\left\lceil\log _{W \times E} \frac{N_{\text {data }}}{P}\right\rceil$, where $N_{\text {data }}$ is the number of data values to be sorted, $P$ is the width of the sorting network, $W$ is the number of leaves in the virtual merge sorter tree, and $E$ is the number of input ports in the high-bandwidth merge sorter tree. In addition, theoretical peak throughput of the sorting engine can be formulated as $\frac{E \times B \times F}{N_{\text {pass }}}$, where $F$ is the operating frequency and $B$ is the data size of a data value. Please note that this formula is based on the assumption that the memory bandwidth is infinite and the active rate [7] is 1.

\section{CREATING AN OPENCL LIBRARY FOR DATA SORTING}

As mentioned in Section 1, we focus on providing the sorting engine to application developers so that they can develop their FPGA codes easily, and we believe that OpenCL programming model for FPGA is currently the best solution for that purpose. In this section, we describe how the RTLtuned sorting engine can be used in an OpenCL programming environment.

The Intel FPGA SDK for OpenCL includes a feature [11] that enables the user's own RTL modules to be an OpenCL library that can be called from the OpenCL kernel code. We briefly explain the programming model of the Intel FPGA SDK for OpenCL using the generated OpenCL library. The kernel code that includes the OpenCL library is compiled using an Intel FPGA OpenCL compiler, which is offered by the Intel FPGA SDK for OpenCL. The compiler is used to convert into synthesizable Verilog HDL files, which are then used in Quartus Prime to generate an .aocx file that includes FPGA configuration information. The host code is for programming the host application; it runs on a host PC and manages an FPGA device at runtime using a set of common application programming interfaces (APIs). To generate a 
Table 1: Comparison results between our sorting library $(W=4, P=8$, and $E=4)$ and the restructured merge sort algorithm [12] for $2^{29}$ data values. The resource usage excludes resources for the OpenCL BSP.

\begin{tabular}{c|cc|cc|rr|rr|r|r}
\hline \hline & ALMs & (\%) & Registers & $(\%)$ & M20Ks & (\%) & DSPs & $(\%)$ & fmax [MHz] & Throughput [MB/s] \\
\hline Our sorting library & 86,856 & $9 \%$ & 244,697 & $7 \%$ & 486 & $4 \%$ & 2 & $0.03 \%$ & 309.98 & 16 \\
The merge sort [12] & 35,455 & $4 \%$ & 73,736 & $2 \%$ & 231 & $2 \%$ & 0 & $0 \%$ & 283.60 & 0.26 \\
\hline
\end{tabular}

host binary, the code is compiled using a standard C compiler such as GCC on Linux, and its object file is linked to the Intel FPGA SDK for OpenCL runtime libraries. At a runtime of the host application, the .aocx file is downloaded to the FPGA using the OpenCL APIs; any data required for kernel execution and any data generated are transferred via the PCIe bus.

Reference [11] presents an overview of the library creation process that contains the sorting function. First, an intermediate object file (. aoco file) is generated from the XML file that defines the configuration of our sorting engine using the aoc -c command. The parameter values of $W, P$, and $E$ described in Section 3.4 are defined in this XML file, and application developers can determine the optimal configuration of the sorting engine while considering the amount of target FPGA resources and required sorting performance. Then, an OpenCL library (.aoclib file) that contains the sorting function is created from the generated . aoco file using the aocl library create command.

An OpenCL kernel code snippet with the sorting library is shown in Figure 6. Initially, it is necessary to include the header file (fpga_sort. $h$ ) that declares the signatures of the sorting function offered from the engine. The sorting function in the library is called in Line 11 and requires three arguments: tmp, src, and numdata. As described in Section 3.4, our sorting engine requires two memory areas because reading and writing data from/to memory occur simultaneously, and initial data values are stored in an $\mathrm{src}[]$ array. These arguments are sent to the sorting engine by the Avalon-ST protocol, and it starts the sorting process. Access to the external memory is performed through the AvalonMM interface using the address to which the pointer pointed and that is passed by the arguments as the base address. Please note that the Intel FPGA SDK for OpenCL does not allow you to pass local memory pointers to RTL modules that can be called in the OpenCL kernel code. When the sorting process is complete, the sorting engine returns a value indicating in which array $(\operatorname{tmp}[]$ or $\operatorname{src}[])$ the sorted data values are stored.

\section{EVALUATION}

We implemented the sorting engine in Verilog HDL and created the OpenCL library that contains the sorting function

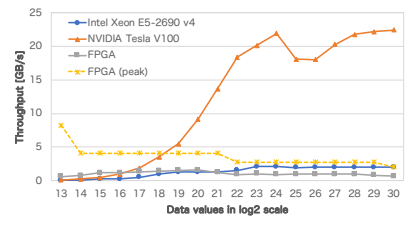

Figure 7: Comparison of sorting performances based on data size.

with Intel FPGA SDK for OpenCL, the version for which was 19.4.0 Build 64 Pro Edition. Our solution was deployed on a BittWare $520 \mathrm{~N}$ board featuring an Intel Stratix 10 FPGA (1SG280HN2F43E2VG). In this study, we focus on the fact that the sorting engine can be used in OpenCL kernel code as an OpenCL library and, therefore, we evaluated its hardware resource usage and sorting performance itself as offered from the OpenCL library. This means that this evaluation did not consider data transfer between the CPU and FPGA. For this evaluation, we used 64-bit data values composed of 32-bit payload fields and 32-bit key fields. The keys used in this evaluation were positive integers.

We first compared the proposed sorting library with a sorting algorithm that assumes the use of HLS. Parallel Programming for FPGAs [12] introduced a merge sort algorithm restructured for the Xilinx Vivado HLS and we reimplemented it for the Intel FPGA SDK for OpenCL. Table 1 shows the comparison results. We used $2^{29}$ data values that were randomly generated and uniformly distributed. The data size was determined according to our target application (i.e., regular path query against large labeled graphs [13] in which sort plays a crucial role in improving the overall performance). The parameter values of $W, P$, and $E$ for the sorting engine were the same as those described in Section 3.4. Our sorting library consumes more than twice the overall hardware resources compared to merge sort [12], but its operating frequency is $1.09 \mathrm{x}$ higher and its sorting throughput is three orders of magnitude greater. Normally, our sorting library itself can operate at $478.01 \mathrm{MHz}$, but a control logic for global memory interleave that is automatically generated by the aoc has become a critical path. It can be removed by disabling global memory interleave and selecting a ring topology as a global memory interconnection by means of flags in the aoc as described in [2]. Through this application, the fmax became $378.21 \mathrm{MHz}$, which was close to the maximum operating frequency of $380 \mathrm{MHz}$ achievable by the actual combination of the OpenCL toolchain and Board Support Package (BSP) offered from BittWare, and the sorting performance was further improved.

Second, we compared the sorting performances of the FPGA and those of a CPU and GPU. In this comparison, we used the parameters $W=64, P=32$, and $E=4$, which typically 
achieve the highest performance and efficiency. Figure 7 shows the comparison results. The CPU performance was assessed by compiling the OpenMP-versioned radix sort based on [14] using Intel C++ Compiler 18.0.1 with -03 and -xHost options, and the GPU performance was assessed by using the Thrust library (CUDA 9.2.148). The FPGA (peak) was derived using the performance model described in Section 3.4, and the effective performance of the FPGA showed the same performance trend. It was observed that the sorting performance of the FPGA for data of small size was better than those of the other two devices. This was because the sorting engine is a pipelined architecture, and the effect of data size is minimal. In addition, the data size is too small for the number of computing cores of the CPU and GPU (particularly the GPU). The ability to process small problem sizes at high speed is necessary to achieve strong scaling for FPGA-centric applications, and sorting is required in nearly all algorithms, particularly in data intensive applications (e.g., statistics (percentile), search (k-nearest neighbor query), index construction (inverted index), and our target (graph processing)). Therefore, we believe that our study is worthwhile for developing a hardware logic to accelerate that fundamental process and enabling its ease of use as a library. It is also critical to estimate the performance of the entire application in advance using the performance model derived in this study and to use the most suitable device for the application in the right place at the right time, while considering the nature and extent of the sorting process in terms of the data size for the entire application.

\section{CONCLUSION}

In this study, we proposed a sorting library that can be used with the OpenCL programming model for FPGA, which is built by combining three hardware sorting algorithms. Our sorting library consumes more than twice the overall hardware resources compared to the merge sort restructured for the OpenCL programming model for FPGA. However, its operating frequency is $1.09 \mathrm{x}$ higher and its sorting throughput is three orders of magnitude greater. We also derived the performance model for data sorting to assist application developers in determining the optimal configuration of the sorting engine while considering the number of target FPGA resources and the required sorting performance.

\section{ACKNOWLEDGMENTS}

This work was supported in part by the "Next Generation High-Performance Computing Infrastructures and Applications R\&D Program" (Development of Computing Communication Unified Supercomputer in Next Generation) of MEXT. This research was also supported in part by the Multidisciplinary Cooperative Research Program in CCS, University of Tsukuba, and JSPS KAKENHI Grant Number 19K20276. We also thank the Intel University Program for providing hardware and software.

\section{REFERENCES}

[1] Norihisa Fujita, Ryohei Kobayashi, Yoshiki Yamaguchi, Tomohiro Ueno, Kentaro Sano, and Taisuke Boku. Performance evaluation of pipelined communication combined with computation in opencl programming on fpga. In 2020 IEEE International Parallel and Distributed Processing Symposium Workshops (IPDPSW), pp. 450-459, 2020.

[2] P. Gorlani, T. Kenter, and C. Plessl. Opencl implementation of cannon' s matrix multiplication algorithm on intel stratix 10 fpgas. In 2019 International Conference on Field-Programmable Technology (ICFPT), pp. 99-107, 2019.

[3] Donald E. Knuth. The Art of Computer Programming, Volume 3: (2Nd Ed.) Sorting and Searching. Addison Wesley Longman Publishing Co., Inc., Redwood City, CA, USA, 1998.

[4] Dirk Koch and Jim Torresen. Fpgasort: A high performance sorting architecture exploiting run-time reconfiguration on fpgas for large problem sorting. In Proceedings of the 19th ACM/SIGDA International Symposium on Field Programmable Gate Arrays, FPGA '11, p. 45-54, New York, NY, USA, 2011. Association for Computing Machinery.

[5] Jared Casper and Kunle Olukotun. Hardware acceleration of database operations. In Proceedings of the 2014 ACM/SIGDA International Symposium on Field-Programmable Gate Arrays, FPGA '14, p. 151-160, New York, NY, USA, 2014. Association for Computing Machinery.

[6] M. Saitoh, E. A. Elsayed, T. V. Chu, S. Mashimo, and K. Kise. A highperformance and cost-effective hardware merge sorter without feedback datapath. In 2018 IEEE 26th Annual International Symposium on Field-Programmable Custom Computing Machines (FCCM), pp. 197204, 2018.

[7] M. Saitoh and K. Kise. Very massive hardware merge sorter. In 2018 International Conference on Field-Programmable Technology (FPT), pp. 86-93, 2018.

[8] T. Usui, T. Van Chu, and K. Kise. A cost-effective and scalable merge sorter tree on fpgas. In 2016 Fourth International Symposium on Computing and Networking (CANDAR), pp. 47-56, 2016.

[9] K. Manev and D. Koch. Large utility sorting on fpgas. In 2018 International Conference on Field-Programmable Technology (FPT), pp. 334-337, 2018.

[10] K. E. Batcher. Sorting networks and their applications. In Proceedings of the April 30-May 2, 1968, Spring Joint Computer Conference, AFIPS '68 (Spring), pp. 307-314, New York, NY, USA, 1968. ACM.

[11] Intel FPGA SDK for OpenCL. https://www.intel.com/content/dam/ www/programmable/us/en/pdfs/literature/hb/opencl-sdk/archives/ aocl_programming_guide-19-4.pdf.

[12] R. Kastner, J. Matai, and S. Neuendorffer. Parallel Programming for FPGAs. ArXiv e-prints, May 2018.

[13] Isabel F. Cruz, Alberto O. Mendelzon, and Peter T. Wood. A graphical query language supporting recursion. In Proceedings of the 1987 ACM SIGMOD International Conference on Management of Data, SIGMOD '87, p. 323-330, New York, NY, USA, 1987. Association for Computing Machinery.

[14] Nadathur Satish, Changkyu Kim, Jatin Chhugani, Anthony D. Nguyen, Victor W. Lee, Daehyun Kim, and Pradeep Dubey. Fast sort on cpus and gpus: A case for bandwidth oblivious simd sort. In Proceedings of the 2010 ACM SIGMOD International Conference on Management of Data, SIGMOD '10, p. 351-362, New York, NY, USA, 2010. Association for Computing Machinery. 